\title{
"WutS" Wundüberwachungssystem mit textiler Sensorik
}

\author{
M. Hoerr ${ }^{1}$, C. Neusser ${ }^{1}$, N. Kasischke ${ }^{2}$, F. Schmitt ${ }^{2}$, T. Gries ${ }^{1}$ und S. Jockenhoevel ${ }^{1}$ \\ ${ }^{1}$ Institut für Textiltechnik, RWTH Aachen Universität, Aachen, Deutschland \\ ${ }^{2}$ BYTEC Medizintechnik GmbH, Eschweiler, Deutschland \\ Kontakt: melanie.hoerr@ita.rwth-aachen.de
}

\begin{abstract}
Zusammenfassung
Im Rahmen des ZIM-Kooperationsprojektes „WutS - Wundüberwachungssystem mit textiler Sensorik“ werden textilintegrierte, gestickte Sensoren für Wundauflagen zur Messung von Temperatur und Feuchte, um Entzündungsvorgänge frühzeitig zu erkennen, entwickelt. Dabei werden innovative Kombinationsansätze aus textilbasierten Sensoren und medizintauglichen Auswertungsalgorithmen entwickelt, die eine neuartige Wundüberwachung ermöglichen. Diese sind die Basis für eine kontinuierliche und den Bedürfnissen von Patienten und medizinischem Fachpersonal entsprechende Wundüberwachung. Durch die Möglichkeit einer kontinuierlichen Überwachung des Zustandes der Wunde ohne Verbandwechsel kann anhand einer neuartigen funktionalisierten und personalisierten Wundauflage ein zu häufiger Verbandwechsel vermieden werden. Dadurch werden sowohl formell als auch informell Pflegende erheblich unterstützt und die Belastung der Patienten reduziert. Komplikationen können frühzeitig erkannt und dementsprechend behandelt werden. Die Integration von textiler Sensorik in Wundauflagen hat den großen Vorteil, dass diese auch mit integrierter Sensorik unverändert weich und geschmeidig bleiben, da keine größeren „Fremdkörper" (Sensoren klassischer Bauart) zusätzlich integriert werden müssen. Weiterhin ist diese im Vergleich hohe erreichbare Integrationstiefe wesentlicher Vorteil des Konzepts und erlaubt eine sehr wundnahe Erfassung physikalischer Parameter.
\end{abstract}

Schlagworte: Wundüberwachung, Wundmanagement, Textile Sensorik, Sticken

\section{Einleitung}

Auf dem Gebiet der Wundversorgung werden erhebliche Gesundheitskosten erzeugt. Im Jahr 2008 wurden ca. 11,2 Mio. Operationen in Krankenhäusern durchgeführt. Die anschließende Wundversorgung wurde bei ca. 9 Mio. Patienten ambulant durchgeführt [1]. Zusätzlich sind jährlich etwa 4 Mio. Patienten von chronischen Wunden betroffen [2].

Die Überwachung des Wundstatus und die sich dadurch ableitende Behandlungskonsequenz stellt ein wichtiges Instrument zur bedarfsgerechten Wundversorgung dar. Aufgrund eines zunehmenden Missverhältnisses zwischen Patientenzahl und betreuendem Fachpersonal als Folge des demographischen Wandels gestaltet sich die patienten-optimierte (Wund-)Versorgung der Patienten zunehmend schwierig.

Die Integration von Sensoren in Wundauflagen, welche Informationen zum Wundheilungsprozess und/oder pathologischen
Prozessen in der Wunde liefern, erleichtert die patienten-optimierte Behandlung von Wunden insbesondere in der häuslichen Pflege. Hierbei spielt die kostengünstige Produktion und die einfache Handhabung im Alltag eine entscheidende Rolle.

\section{Methoden und Materialien}

Leitfähige, faserförmige Materialen können durch die Sticktechnologie kostengünstig auf Wundauflagen aufgebracht werden. Durch die geeignete Auswahl des Fasermaterials und insbesondere einer spezifischen Anordnung der Materialien auf der Wundauflage, können durch die Kombination mit geeigneter miniaturisierter Messtechnik Sensorsysteme entwickelt werden. Dieses Sensorsystem kann zur gleichzeitigen Messung der Temperatur und der Feuchtigkeit in der Wunde verwendet werden. Durch ein solches textilintegriertes Sensorsystem wird eine patientenindividuelle Therapie ermöglicht, ohne die Eigenschaften der textilen Wundauflage zu beeinträchtigen. 
Hierzu werden unterschiedliche leitfähige faserförmige Materialien mit Hilfe der Sticktechnologie in eine Wundauflage integriert. Insbesondere das leitfähige Material, welches in die Wundauflage integriert wird, hat einen wesentlichen Einfluss auf die textilen Eigenschaften der Wundauflagen. Hierzu werden silberplattierte Kupferlackdrähte der Elektrisola Dr. Gerd Schildbach $\mathrm{GmbH}$ \& Co. KG, Reichshof-Eckenhagen, auf ihre Eignung als Sensorfühler untersucht. Silberplattierter Kupferdraht der Temperaturklasse $155^{\circ} \mathrm{C}$ mit einer Grad 1 Lackschicht werden mit unterschiedlichen Durchmessern auf ihre Eignung als Sensorfühler in einer Wundauflage hin untersucht, vgl. Tab. 1. Silberplattierter Kupferdrähte bestehen aus einem Kupferkern mit konzentrischer Silberplattierung mit einem Anteil von $50 \mathrm{~g}$ Silber pro kg Drahtgewicht. Diese Drähte weisen eine sehr gute Lötbarkeit und hohe thermische Werte aus. Das Material weißt einen Temperaturkoeffizient des elektrischen Widerstandes von $41000 * 10 \mathrm{E}-6 / \mathrm{K}$ auf.

Tab. 1: Materialauswahl für textile Sensorfühler aus versilberten, lackierten Kupferfeindraht/-fasern

\begin{tabular}{|c|c|c|}
\hline $\begin{array}{c}\text { Nenn- } \\
\text { durchmesser } \\
\text { in } \mathbf{~ m m}\end{array}$ & $\begin{array}{c}\text { Schichtdicke } \\
\text { mod. } \\
\text { Polyurethan } \\
\text { in } \boldsymbol{\mu m}\end{array}$ & $\begin{array}{c}\text { Nom. } \\
\text { Widerstand } \\
\text { bei } \mathbf{2 0}^{\circ} \mathbf{C} \text { in } \\
\mathbf{O h m} / \mathbf{m}\end{array}$ \\
\hline 0,071 & $7-13$ & 4,318 \\
\hline 0,100 & $9-16$ & 2,176 \\
\hline 0,140 & $11-20$ & 1,110 \\
\hline
\end{tabular}

Eine typische Spannungs-Dehnungskurve dieses Feindrahtes am Beispiel des Durchmessers $0.05 \mathrm{~mm}$ ist in Abb. 1 dargestellt.

Tensile strength vs. elongation (Diameter: 0.050mm)

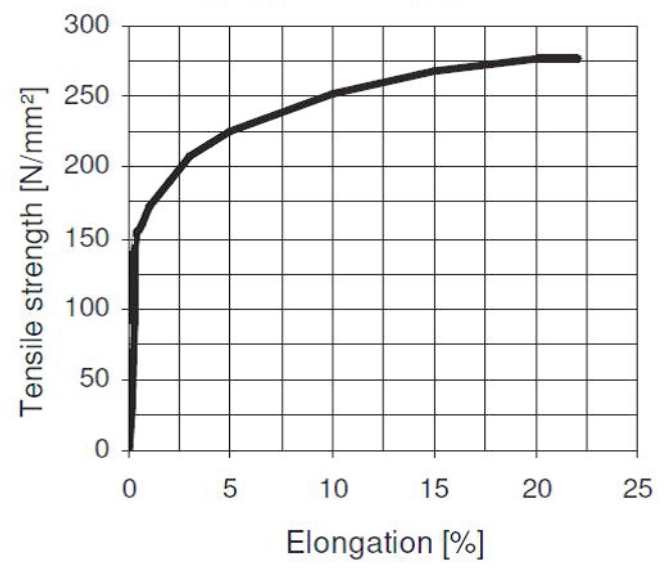

Abb. 1: Spannungs-Dehnungs-Diagramm für Elektrisola silberplattierter Kupferdraht bei einem Nenndurchmesser von $0.050 \mathrm{~mm}$
Die Applikation der Feindrähte auf die Wundauflage kann mittels zweier unterschiedlicher Sticktechnologien erfolgen.

\section{Tailored Wire Placement Drahtverlegemethode}

Bei der Drahtverlegetechnolgie, der s.g. Tailored Wire Placement Methode, wird der Draht während des Stickprozesses auf das Textil aufgelegt und mittels eines Zick-ZackStiches fixiert. Die Prinzipdarstellung ist in Abb. 2 dargestellt.

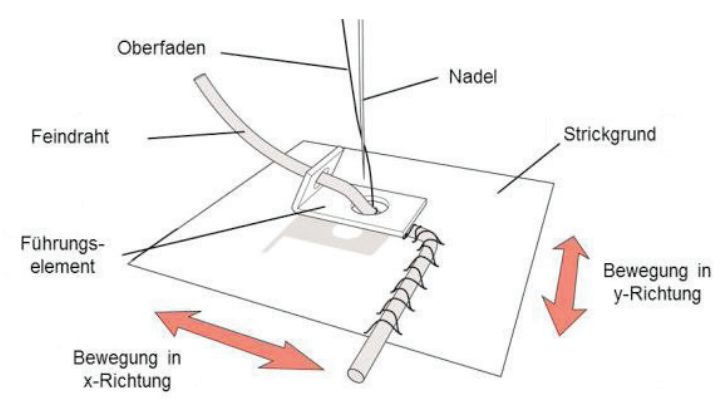

Abb. 2: Prinzip der Tailored Wire Placement Verlegemethode zur Applikation des Feindrahtes

\section{Doppelsteppstichsticken von Draht}

Bei der Doppeltsteppstichstickerei (DSSStickerei) handelt es sich um die konventionell am häufigsten eingesetzte Sticktechnologie. Hierbei werden durch einen dem Nähverfahren ähnlichen Prozessablauf ein Ober- und Unterfaden so miteinander verschlauft, dass auf der Oberseite des Trägermaterials eine Applikation aufgestickt wird. Diese Technologie wird z.B. zum Sticken von Logos oder Schriftzügen eingesetzt. In der konventionellen Stickerei wird als Oberfaden ein Farb- oder Effektgarn als „Funktionsgarn“ eingesetzt, um eine modische Applikation auf dem Textil herzustellen. Als Unterfaden wird in der Regel ein neutrales weißes Stickgarn eingesetzt, dessen Aufgabe die Schlaufenbildung mit dem Oberfaden ist, was notwendig ist um einen Stich zu erzeugen.

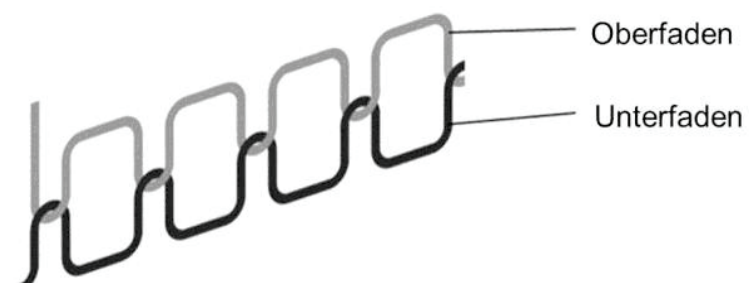

Abb. 3: Stichbild Doppelsteppstich mit mittiger Verschlaufung von Ober- und Unterfaden

Zur Applikation eines Drahtes mittels dieser Sticktechnologie wird dieses Prinzip umgedreht. Der Oberfaden übernimmt hier nun die Aufgabe der Schlaufenbildung. Hierfür können konventionelle Stickgarne verwendet werden. Der Unterfaden wird in diesem Fall durch den Draht gebildet und liegt nahezu gestreckt auf 
der Unterseite des Trägermaterials vor. Der Oberfaden bindet den Draht an den Positionen der Stiche ab und hält inn somit in Position.

Die geometrische Anordnung der Sensorfühler, die bei der sensorisierten Wundauflage aus aufgestickten Drahtfasern bestehen, hat einen erheblichen Einfluss auf die Messergebnisse. Je nach geometrischer Anordnung der Sensorfühler können unterschiedliche oder auch mehrere Werte gleichzeitig gemessen werden. In Abb. 4 bis Abb. 6 sind drei mögliche unterschiedliche Geometrie zur Ermittlung der Messwerte dargestellt.

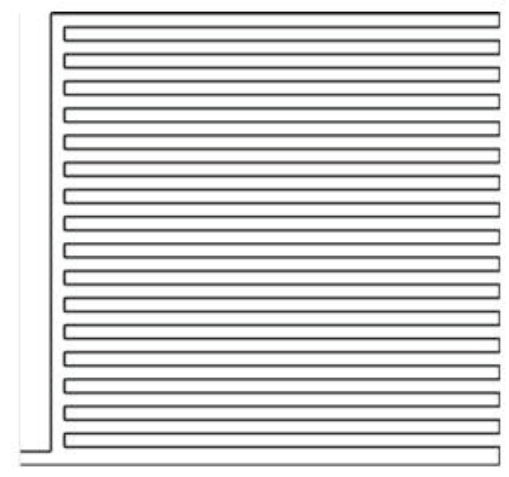

Abb. 4: Mäanderstruktur zur Temperaturmessung

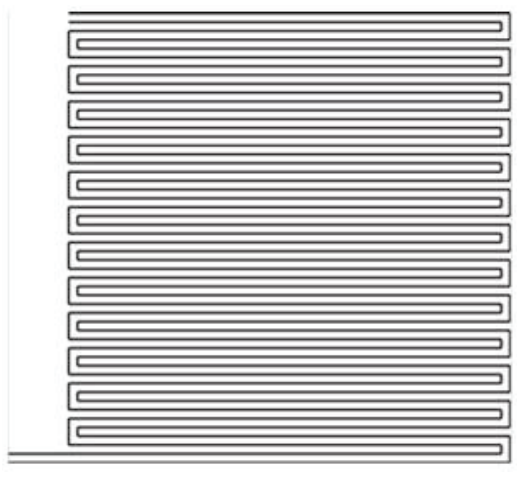

Abb. 5: Doppelmäander zur Feuchtemessung

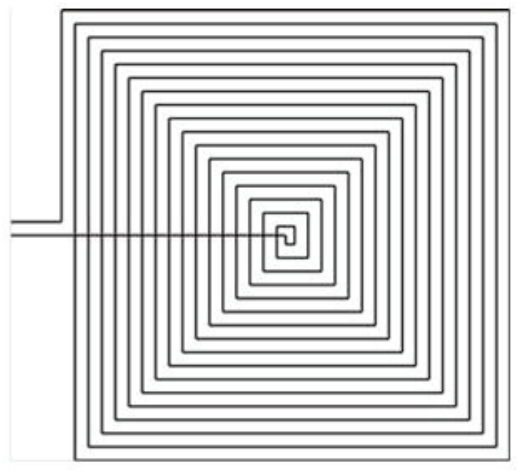

Abb. 6: Spirale zur Dehnungsmessung

Jede der in Abb. 4 bis Abb. 6 abgebildeten Geometrie kann mit unterschiedlichen Abständen (min. 0,5 m) zwischen den Drahtfasern abgebildet werden. Die Größe der Abstände hat einen erheblichen Einfluss auf die
Messgenauigkeit, aber auch gleichzeitig auf die Drapierbarkeit und somit auf den Komfort der sensorisierten Wundauflage.

\section{Ergebnisse}

Zur Evaluierung der Ergebnisse werden folgende Kriterien zu Grunde gelegt:

Verstickbarkeit

Die Verstickbarkeit ist die wichtigste Eigenschaft zur Charakterisierung der Eignung eines fadenförmigen Materials. Sie gibt Auskunft über das Fadenbruchverhalten des Drahtes während des Stickprozesses. Falls der Draht während des Stickprozesses bricht oder reißt, kann der Sensor nicht verwendet werden, dies wiederum führt zu einem erhöhten Ausschuss. Drähte, die aufgrund einer hohen Fadenbruchneigung zu hohem Ausschuss an Sensoren führen, sind für die Anwendung als textile Sensoren in der Wundüberwachung nicht geeignet. Ein weiteres Evaluationskriterium, welches ebenfalls der Verstickbarkeit zuzuordnen ist, ist die Ebenmäßigkeit der Applikation des Drahtes auf dem Trägertextil. Eine ebenmäßige Applikation zeigt sich durch eine gleichmäßige Stichbildung des Drahtes ohne Schlaufenbildung ober- oder unterhalb des Trägertextiles. Bei der Entstehung von Schlaufen ober- oder unterhalb des Trägertextiles kann es zu einer Verfälschung der Messergebnisse kommen. Aus diesem Grunde ist die Ebenmäßigkeit der Applikation ein wichtiger Indikator für die spätere Qualität des Sensors.

Biege- und Drapierverhalten

Das Biege- sowie Drapierverhalten der sensorisierten Wundauflage sind wichtige Evaluierungskriterien hinsichtlich der Einsatzmöglichkeiten. Bei der Integration der Sensorfühler in die Trägerstruktur ist darauf zu achten, dass die textilen Eigenschaften der Wundauflage nicht beeinträchtigt werden. Hierfür geben Biege- und Drapierfähigkeit gute quantifizierbare Ergebnisse. Zum einen dürfen die Messergebnisse durch Biegung nicht verfälscht werden und zum anderen ist ein Brechen des Drahtes aufgrund von Biegung und Drapierung unbedingt auszuschließen. Die Feinheit des Drahtes hat hierdrauf einen sehr wesentlichen Einfluss; dünne Drähte beeinflussen die textilen Eigenschaften geringer, weisen jedoch gleichzeitig ein höheres Bruchrisiko bei Biegung auf.

Messfeinheit und Auflösung

Die Messfeinheit und Auflösung der Sensorwundauflage steht in Abhängigkeit zum Material, welches für die Sensorfühler 
verwendet wird. Auch wenn insbesondere hinsichtlich dieses Kriteriums die Messelektronik und die Algorithmen einen entscheidenden Part spielen, kann die Elektronik nur Messwerte ermitteln, die von den Sensorfühlern ermittelt werden können. Hierdurch werden die Genauigkeit der Messwerte und die Abweichungen bestimmt.

\section{Diskussion}

Zur Kontrolle des Heilungsverlaufes einer Wunde sind die Parameter Temperatur, Feuchte und Schwellung wichtige Indikatoren. Hierbei steht das Wohl des Patienten im Vordergrund. Daher ist es unablässig, dass durch die Sensorintegration keine zusätzliche Reizung der Wunde oder eine Mehrbelastung des Patienten geschieht. Um dies zu erreichen und die benötigten Messwerte in einer Wundauflage messen zu können, ist es wichtig, die richtige Materialauswahl $\mathrm{zu}$ treffen und dieses mit der geeigneten Technologie zu integrieren. Des Weiteren ist die Anordnung der Sensorfühler auf der Wundauflage ein wichtiger Einflussfaktor auf das Ergebnis der Sensorwerte. Hierbei sind sowohl Material- als auch technologische Grenzen zu beachten. So können z.B. mit der Verlegetechnologie die Drahtfasern nicht näher als $1,5 \mathrm{~mm}$ nebeneinander abgelegt werden, da durch den Zick-Zack-Stich zur Fixierung neben den Drahtfasern jeweils noch genügend Platz für die Nadel bleiben muss. Bei der DSS-Stickerei kann dieser Abstand auf $0,5 \mathrm{~mm}$ verringert werden, da hier eine Nadelbreite in der Mitte der Drahtfaser ausreichend ist. Dies hat zur Folge, dass die Sticktechnologie in Abhängigkeit der Sensorgeometrie steht und umgekehrt.

Aufgrund der vielseitigen Möglichkeiten der unterschiedlichen Sticktechnologien und der Möglichkeiten der Verwendung unterschiedlicher feinster Drahtfasern, die im Durchmesserbereiches eines Haares liegen, ist es möglich, sehr feine Strukturen auf eine Wundauflage aufzusticken, die als Sensorfühler eine Wundüberwachung ermöglichen. In folgenden Untersuchungen werden die Grenzen der Dimensionierung ermittelt und die Evaluierung der Wundauflage vorgenommen.

\section{Literatur}

[1] Zahlen des statistischen Bundesamtes, 2008

[2] JANSSEN H. et al.: Kosten-NutzenBewertung in der Versorgung chronischer Wunden Clusteranalyse nach Dauer der Behandlung und Kostentypologie Institut für Gesundheits- und Pflegeökonomie (IGP), Hochschule Bremen, Wund Management 01/2011

\section{Danksagung}

Für die finanzielle Unterstützung des ZIMProjektes, KF3414615AJ4, sei dem Bundesministerium für Wirtschaft und Energie aufgrund eines Beschlusses des Deutschen Bundestages, gedankt. 